\title{
Erratum to: Fourier Transform Ion Cyclotron Resonance Mass Resolution and Dynamic Range Limits Calculated by Computer Modeling of Ion Cloud Motion
}

Gleb Vladimirov, ${ }^{1,2,3}$ Christopher L. Hendrickson, ${ }^{4,5}$ Greg T. Blakney, ${ }^{5}$ Alan G. Marshall, ${ }^{4,5}$ Ron M. A. Heeren, ${ }^{6}$ Eugene N. Nikolaev ${ }^{1,2,3}$

${ }^{1}$ The Institute for Energy Problems of Chemical Physics, Russian Academy of Sciences, Moscow, Russia

${ }^{2}$ Emanuel Institute of Biochemical Physics of Russian Academy of Sciences, Moscow, Russia

${ }^{3}$ Institute of Biomedical Chemistry of Russian Academy of Medical Sciences, Moscow, Russia

${ }^{4}$ Department of Chemistry and Biochemistry, Florida State University, Tallahassee, FL, USA

${ }^{5}$ Ion Cyclotron Resonance Program, National High Magnetic Field Laboratory, Tallahassee, FL, USA

${ }^{6}$ FOM Institute for Atomic and Molecular Physics (AMOLF), Amsterdam, The Netherlands

Erratum to: J. Am. Soc. Mass Spectrom

DOI: $10.1007 / \mathrm{s} 13361-011-0268-8$

Additional affiliations for Gleb Vladimirov and Eugene N. Nikolaev were omitted. Author affilliations are complete and corrected above. 